\title{
Finite-temperature effective field theory for dark solitons in superfluid Fermi gases
}

\author{
S. N. Klimin, ${ }^{*}$ J. Tempere, ${ }^{\dagger}$ and J. T. Devreese ${ }^{\ddagger}$ \\ TQC, Universiteit Antwerpen, Universiteitsplein 1, B-2610 Antwerpen, Belgium
}

(Received 11 July 2014; published 11 November 2014)

\begin{abstract}
We use a finite-temperature effective field theory recently developed for superfluid Fermi gases to investigate the properties of dark solitons in these superfluids. Our approach provides an analytic solution for the dip in the order parameter and the phase profile across the soliton, which can be compared with results obtained in the framework of the Bogoliubov-de Gennes equations. We present results in the whole range of the BCS-BEC crossover, for arbitrary temperatures and taking into account Gaussian fluctuations about the saddle point. The obtained analytic solutions yield an exact energy-momentum relation for a dark soliton showing that the soliton in a Fermi gas behaves like a classical particle even at nonzero temperatures. The spatial profile of the pair field and for the parameters of state for the soliton are analytically studied.
\end{abstract}

DOI: 10.1103/PhysRevA.90.053613

PACS number(s): 67.85.Lm, 67.85.De, 03.75.Ss

\section{INTRODUCTION}

The recent progress in the experimental and theoretical study of quantum gases has been particularly stimulated by the fact that they represent an example of macroscopic quantum phenomena where the system parameters can be finely tuned. For Fermi gases, a crossover between the Bardeen-CooperSchrieffer (BCS) superfluid constituted by Cooper pairs and a Bose-Einstein condensate (BEC) of bound fermion pairs can be achieved using the Feshbach resonance. Nonlinear phenomena in ultracold atomic gases, especially relating to vortices and solitons, draw great interest, as they provide insight into the interplay of interactions and coherence $[1,2]$. A dark soliton in a superfluid is a nonlinear solitary excitation of the order parameter which propagates on a uniform or plane-wave background and manifests itself through a density dip. Dark solitons are one of the first fundamental nonlinear excitations that have been experimentally detected in BECs [3,4]. In addition to ultracold gases, there have been experimental observations of dark solitons in other systems, including optical [5] and mechanical [6,7] dark solitons. However, the experimental realization of dark solitons in atomic ${ }^{6} \mathrm{Li}$ Fermi gases near a Feshbach resonance occurred only recently [8]. The study of these dark solitons in Fermi gases, especially with respect to the snake instability and the subsequent decay into vortex filaments or rings, is a subjects of active current debate [9-12].

Dark solitons in the BECs were successfully treated theoretically using the Gross-Pitaevskii (GP) equation [13], both within the mean-field approximation $[4,14]$ and taking into account quantum and thermal fluctuations [15-19]. For the theoretical description of solitons in superfluid Fermi gases in the BCS-BEC crossover regime, however, the GP equation appears not to be applicable, except in the deep BEC limit, where the pairs can be approximately considered as a Bose gas

\footnotetext{
*sergei.klimin@uantwerpen.be; Also at Department of Theoretical Physics, State University of Moldova, 2009 Chişinău, Moldova.

${ }^{\dagger}$ Also at Lyman Laboratory of Physics, Harvard University, Cambridge, Massachusetts 02138, USA.

${ }^{\ddagger}$ Also at Technische Universiteit Eindhoven, 5612 AZ Eindhoven, The Netherlands.
}

of molecules. For solitons within the BCS-BEC crossover, one of the most reliable methods is based on the Bogoliubov-de Gennes (BdG) equations (see review in [20]). However, solving the BdG equations is computationally very demanding, due to the necessity of using a large amount of fermionic wave functions. As a result, analysis of the BdG solutions for dark solitons, at least at present, has been performed only in the zero-temperature case $[11,21,22]$. Computationally less demanding extensions of the $\mathrm{BdG}$ approach based on coarsegraining have been developed recently [23] but not yet applied to solitons, because in the present form it is not time dependent. This inspires attempts to develop complementary approaches for ultracold Fermi gases exploiting only a macroscopic wave function. Prominent examples of such attempts are the modifications of the Ginzburg-Landau (GL) approach [24,25] for cold Fermi gases in the BCS-BEC crossover, and the GP and nonlinear Schrödinger equations [26]. The GL method is valid in a rather narrow temperature region close to the critical temperature $T_{c}$. The GP equation works well in the BEC regime but can fail at weaker couplings. There are extensions of the GL approach to lower temperatures based on expanding the free energy in powers of the small parameter $\eta \equiv 1-T / T_{c}$ [27-30] or using a microscopic treatment [31-33]. Our recent investigation [34,35], focused on atomic Fermi gases in the BCS-BEC crossover regime, has been devoted to the development of an effective method for the description of the macroscopic wave function of a fermionic superfluid system without assuming $\eta$ small. In Refs. [34] and [35], the GL formalism has been extended to the whole temperature range below $T_{c}$ for a multiband superfluid fermion system. In the limit $T \rightarrow T_{c}$, the theory from Ref. [24] is retrieved.

In the present work, we apply the effective field theory (EFT) of Ref. [34] to dark solitons in a superfluid Fermi gas with $s$-wave pairing. The fermion system is treated in the BCS-BEC crossover and in the whole range of temperatures below $T_{c}$. The study is performed both within the mean-field approximation and taking into account Gaussian fluctuations in renormalization of the chemical potential of the Fermi gas. The mean-field results are compared with BdG data in the low-temperature limit, allowing us to reliably establish the range of validity of the EFT. 
The paper is organized as follows. In Sec. II, we present the field equations and derive their analytic solution for dark solitons. In Sec. III, the macroscopic integrals of motion for the soliton are calculated, and the exact energy-momentum relation is derived for the soliton. Sec. IV presents a discussion of the numeric results for the parameters of the dark soliton, followed by Sec. V, the Conclusions.

\section{FIELD EQUATIONS}

For the analytic treatment of dark solitons in ultracold Fermi gases we use the finite-temperature EFT developed in Refs. [34] and [35]. The description of a Fermi gas with $s$-wave pairing within this formalism is performed using the effective bosonic action for the pair field $\Psi(r, \tau)$ (the macroscopic order parameter). Details of the approach are thoroughly described in Ref. [34]. The key approximation of EFT is the local gradient expansion of the effective field action. We start from the Euclidean-time form of the effective field action from Ref. [35],

$$
S(\beta)=\int_{0}^{\beta} d \tau \int d \mathbf{r}\left[\frac{D}{2}\left(\bar{\Psi} \frac{\partial \Psi}{\partial \tau}-\frac{\partial \bar{\Psi}}{\partial \tau} \Psi\right)+\mathcal{H}\right],
$$

where $\beta$ is the inverse to the temperature and $\mathcal{H}$ is the Hamiltonian of the pair field,

$$
\mathcal{H}=\Omega_{s}+\frac{C}{2 m}\left|\nabla_{\mathbf{r}} \Psi\right|^{2}-\frac{E}{2 m}\left(\nabla_{\mathbf{r}}|\Psi|^{2}\right)^{2}
$$

with the field-dependent thermodynamic potential,

$$
\begin{aligned}
\Omega_{s}= & -\int \frac{d \mathbf{k}}{(2 \pi)^{3}}\left[\frac{1}{\beta} \ln \left(2 \cosh \beta E_{\mathbf{k}}+2 \cosh \beta \zeta\right)\right. \\
& \left.-\xi_{\mathbf{k}}-\frac{m|\Psi|^{2}}{k^{2}}\right]-\frac{m|\Psi|^{2}}{4 \pi a_{s}} .
\end{aligned}
$$

Here, $a_{s}$ is the scattering length for $s$-wave pairing, and $\xi_{\mathbf{k}}=\frac{k^{2}}{2 m}-\mu$ is the kinetic energy of the fermionic atoms with mass $m$, measured from the chemical potential $\mu$. The present formalism can take population imbalance into account by introducing separate chemical potentials for "spin-up" and "spin-down" atoms, combined into $\mu \equiv\left(\mu_{\uparrow}+\mu_{\downarrow}\right) / 2$ and $\zeta \equiv$ $\left(\mu_{\uparrow}-\mu_{\downarrow}\right) / 2 \mu_{\uparrow}$. Finally, $E_{\mathbf{k}}=\sqrt{\xi_{\mathbf{k}}^{2}+|\Psi|^{2}}$ is the Bogoliubov excitation energy. Expression (3) formally coincides with the saddle-point grand-canonical thermodynamic potential for imbalanced Fermi gases [36]. The coefficients in front of the gradients are given by

$$
\begin{gathered}
C=\int \frac{d \mathbf{k}}{(2 \pi)^{3}} \frac{k^{2}}{3 m} f_{2}\left(\beta, E_{\mathbf{k}}, \zeta\right), \\
D=\int \frac{d \mathbf{k}}{(2 \pi)^{3}} \frac{\xi_{\mathbf{k}}}{|\Psi|^{2}}\left[f_{1}\left(\beta, \xi_{\mathbf{k}}, \zeta\right)-f_{1}\left(\beta, E_{\mathbf{k}}, \zeta\right)\right], \\
E=2 \int \frac{d \mathbf{k}}{(2 \pi)^{3}} \frac{k^{2}}{3 m} \xi_{\mathbf{k}}^{2} f_{4}\left(\beta, E_{\mathbf{k}}, \zeta\right) .
\end{gathered}
$$

The functions $f_{s}(\beta, \varepsilon, \zeta)$ are determined through sums over the fermionic Matsubara frequencies $\omega_{n}=(2 n+1) \pi / \beta$ :

$$
f_{s}(\beta, \varepsilon, \zeta) \equiv \frac{1}{\beta} \sum_{n=-\infty}^{\infty} \frac{1}{\left[\left(\omega_{n}-i \zeta\right)^{2}+\varepsilon^{2}\right]^{s}}
$$

For any integer $s$, these sums are analytically calculated, using the recurrence relations given in Ref. [34]:

$$
\begin{gathered}
f_{1}(\beta, \varepsilon, \zeta)=\frac{1}{2 \varepsilon} \frac{\sinh (\beta \varepsilon)}{\cosh (\beta \varepsilon)+\cosh (\beta \zeta)}, \\
f_{s+1}(\beta, \varepsilon, \zeta)=-\frac{1}{2 s \varepsilon} \frac{\partial f_{s}(\beta, \varepsilon, \zeta)}{\partial \varepsilon} .
\end{gathered}
$$

In the limit of low amplitude $|\Psi|$, the gradient term in Hamiltonian (2) with coefficient $C$ is quadratic with respect to $|\Psi|$, and the term with coefficient $E$ is quartic. So, in the vicinity of the critical temperature $T_{c}$ (where the standard GL approach is applicable and $|\Psi|$ is small), this quartic term becomes vanishingly small. Hence it is absent in the standard GL theory, although far below $T_{c}$ it is not negligible.

In order to study the time evolution of ultracold Fermi gas, we need a relation between real-time and Euclidean-time actions. The Euclidean-time action $S(\beta)$ enters the partition function,

$$
\mathcal{Z} \propto \int \mathcal{D}[\bar{\Psi}, \Psi] e^{-S(\beta)},
$$

while the real-time action $S\left(t_{b}, t_{a}\right)$ enters the transition amplitude,

$$
K\left(t_{b}, t_{a}\right)=\int \mathcal{D}[\bar{\Psi}, \Psi] e^{i S\left(t_{b}, t_{a}\right)} .
$$

The correspondence between real-time and Euclidean-time actions is established by the formal replacement in (1):

$$
\tau \rightarrow i t \Leftrightarrow S(\beta) \rightarrow-i S\left(t_{b}, t_{a}\right) .
$$

The real-time action can be then expressed as

$$
S\left(t_{b}, t_{a}\right)=\int_{t_{a}}^{t_{b}} d t \int d \mathbf{r} \mathcal{L},
$$

where $\mathcal{L}$ is the field Lagrangian:

$$
\mathcal{L}=i \frac{D}{2}\left(\bar{\Psi} \frac{\partial \Psi}{\partial t}-\frac{\partial \bar{\Psi}}{\partial t} \Psi\right)-\mathcal{H}
$$

Next, we use the regularized action, subtracting the background thermodynamic potential $\Omega_{s}\left(\left|\Psi_{\infty}\right|\right)$ from the Hamiltonian. Here, $\left|\Psi_{\infty}\right|$ is the modulus of the background order parameter. We express the order parameter through the phase $\theta$ and amplitude $|\Psi|$ as $\Psi=|\Psi| \exp (i \theta)$, using the notation

$$
|\Psi(\mathbf{r}, t)|=\left|\Psi_{\infty}\right| a(\mathbf{r}, t)
$$

where $a(\mathbf{r}, t) \equiv\left|\Psi / \Psi_{\infty}\right|$ is the amplitude modulation function. These notations are suitable for describing localized disturbances such as a vortex or a soliton in an otherwise homogeneous superfluid. For $r \rightarrow \infty, a(r) \rightarrow a_{\infty}=1$. The procedure we follow consists in (i) substituting the above form of $\Psi$ into the Lagrangian, (14), and interpreting the result as the Lagrangian for the amplitude and phase fields, (ii) extracting from it the field equations for $a(\mathbf{r}, t)$ and $\theta(\mathbf{r}, t)$, and (iii) solving these equations.

First, we rewrite the Lagrangian in the amplitude-phase representation,

$$
\mathcal{L}=\int d \mathbf{r}\left(-\kappa(a) a^{2} \frac{\partial \theta}{\partial t}-\mathcal{H}\right)
$$


and the field Hamiltonian becomes

$$
\begin{aligned}
\mathcal{H}= & \int d \mathbf{r}\left[\Omega_{s}(a)-\Omega_{s}\left(a_{\infty}\right)+\frac{1}{2} \rho_{\mathrm{qp}}(a)\left(\nabla_{\mathbf{r}} a\right)^{2}\right. \\
& \left.+\frac{1}{2} \rho_{\mathrm{sf}}(a)\left(\nabla_{\mathbf{r}} \theta\right)^{2}\right] .
\end{aligned}
$$

The coefficient at the time derivative $\kappa$, the quantum pressure coefficient $\rho_{\mathrm{qp}}$, and the superfluid density $\rho_{\mathrm{sf}}$ are determined as follows:

$$
\begin{gathered}
\kappa(a)=D(a)\left|\Psi_{\infty}\right|^{2}, \\
\rho_{\mathrm{sf}}(a)=\frac{C(a)}{m}|\Psi|^{2}, \\
\rho_{\mathrm{qp}}(a)=\frac{C(a)-4|\Psi|^{2} E(a)}{m}\left|\Psi_{\infty}\right|^{2} .
\end{gathered}
$$

They are, in general, dependent on the amplitude $a$. It is easy to verify analytically that the superfluid density given by (19) with (4) is equivalent to the saddle-point superfluid density defined through a "phase twist" on the order parameter, as phase gradients endow the pair condensate with a finite superfluid velocity [37]. The pair condensate also resists gradients in the pair density, $\nabla_{\mathbf{r}} a$, leading to a quantum pressure term as in bosonic condensates.

The stationary wave and soliton solutions propagating at a constant velocity $v_{S}$ obey the relation $f(x, t)=f\left(x-v_{S} t\right)$. The stationary Lagrangian, (16), is then given by

$$
\begin{aligned}
\mathcal{L}= & \int_{-\infty}^{\infty} d x\left\{\kappa(a) a^{2} v_{S} \frac{\partial \theta}{\partial x}-\left[\Omega_{s}(a)-\Omega_{s}\left(a_{\infty}\right)\right]\right. \\
& \left.-\frac{1}{2} \rho_{\mathrm{qp}}(a)\left(\frac{\partial a}{\partial x}\right)^{2}-\frac{1}{2} \rho_{\mathrm{sf}}(a)\left(\frac{\partial \theta}{\partial x}\right)^{2}\right\} .
\end{aligned}
$$

The macroscopic soliton dynamics is determined by the solutions of the Lagrange equations for the Lagrangian, (21), for the phase and amplitude. The equation of motion for the phase reads

$$
\frac{\partial}{\partial x}\left(\rho_{\mathrm{sf}}(a) \frac{\partial \theta}{\partial x}-v_{S} \kappa a^{2}\right)=0 .
$$

The general solution of this equation is

$$
\frac{\partial \theta}{\partial x}=\frac{1}{\rho_{\mathrm{sf}}(a)}\left(C+v_{S} \kappa(a) a^{2}\right),
$$

with the integration constant $C$. Imposing the boundary condition $\partial_{x} \theta \rightarrow 0$ for $x \rightarrow \pm \infty$ corresponds to the "dark soliton" solution, in which the total change of phase accross the soliton is finite. This condition results in the integration constant $C=-v_{S} \kappa_{\infty}$, where $\kappa_{\infty} \equiv \kappa\left(a_{\infty}\right)$, with $a_{\infty} \equiv 1$ the bulk value of the coefficient $\kappa(a)$. Hence the dark soliton solution for the derivative $\partial_{x} \theta$ is

$$
\frac{\partial \theta}{\partial x}=\frac{v_{S}}{\rho_{\mathrm{sf}}(a)}\left[\kappa(a) a^{2}-\kappa_{\infty}\right]
$$

and the phase for the dark soliton can be determined explicitly:

$$
\theta(x)=v_{S} \int_{-\infty}^{x} \frac{\kappa\left(a\left(x^{\prime}\right)\right) a^{2}\left(x^{\prime}\right)-\kappa_{\infty}}{\rho_{\mathrm{sf}}\left(a\left(x^{\prime}\right)\right)} d x^{\prime} .
$$

The total phase change throughout the soliton is determined as the difference

$$
\delta \theta \equiv \theta(-\infty)-\theta(\infty)
$$

and results in the integral,

$$
\delta \theta=v_{S} \int_{-\infty}^{\infty} \frac{1}{\rho_{\mathrm{sf}}(a(x))}\left(\frac{\kappa_{\infty}}{a^{2}(x)}-\kappa(a(x))\right) d x .
$$

The Lagrange equation for the amplitude $a(x)$, with the solution for the phase, (24), takes the form

$$
\begin{aligned}
\frac{\partial}{\partial x}\left(\rho_{\mathrm{qp}} \frac{\partial a}{\partial x}\right)= & \frac{1}{2} \frac{\partial \rho_{\mathrm{qp}}}{\partial a}\left(\frac{\partial a}{\partial x}\right)^{2}+\frac{\partial \Omega_{s}}{\partial a} \\
& -\frac{1}{2} v_{S}^{2} \frac{\partial}{\partial a}\left(\frac{\left[\kappa(a) a^{2}-\kappa_{\infty}\right]^{2}}{\rho_{\mathrm{sf}}(a)}\right) .
\end{aligned}
$$

It also has the exact analytic solution. Imposing the boundary conditions

$$
\left.\frac{\partial a(x)}{\partial x}\right|_{x \rightarrow \pm \infty}=0,\left.\quad a(x)\right|_{x \rightarrow \pm \infty}=1
$$

and introducing the notations

$$
\begin{gathered}
X(a) \equiv \Omega_{s}(a)-\Omega_{s}\left(a_{\infty}\right), \\
Y(a) \equiv \frac{\left[\kappa(a) a^{2}-\kappa_{\infty}\right]^{2}}{2 \rho_{\mathrm{sf}}(a)},
\end{gathered}
$$

we arrive at the symmetric solution for the coordinate $x$ as a function of the relative amplitude $a$ :

$$
x= \pm \frac{1}{\sqrt{2}} \int_{a_{0}}^{a} \frac{\sqrt{\rho_{\mathrm{qp}}\left(a^{\prime}\right)}}{\sqrt{X\left(a^{\prime}\right)-v_{S}^{2} Y\left(a^{\prime}\right)}} d a^{\prime} .
$$

The amplitude modulation function at the soliton center, $a_{0} \equiv$ $a(x=0)$, is determined by the equation

$$
X\left(a_{0}\right)-v_{S}^{2} Y\left(a_{0}\right)=0 .
$$

The exact analytic solutions obtained above for a dark soliton allow us to consider the soliton dynamics in terms of the macroscopic integrals of motion for the soliton: the momentum and the energy. They are determined in the next section through the canonical definitions of the classical Hamilton dynamics in the spirit of Ref. [13].

\section{INTEGRALS OF MOTION}

The total soliton momentum is obtained by differentiating the Lagrangian with respect to the soliton velocity:

$$
\mathcal{P}_{S}^{\text {(tot) }} \equiv \frac{\partial \mathcal{L}}{\partial v_{S}} .
$$

With the Lagrangian (21), the total soliton momentum is

$$
\mathcal{P}_{S}^{\text {(tot) }}=\int_{-\infty}^{\infty} d x \kappa(a) a^{2} \frac{\partial \theta}{\partial x} .
$$

Using the exact solution for the phase, we express the total momentum explicitly:

$$
\mathcal{P}_{S}^{(\mathrm{tot})}\left(v_{S}\right)=v_{S} \int_{-\infty}^{\infty} d x \frac{\kappa(a)}{\rho_{\mathrm{sf}}(a)}\left(\kappa(a)-\frac{\kappa_{\infty}}{a^{2}}\right) .
$$


As established in Ref. [13], the total momentum $\mathcal{P}_{S}^{(\text {tot })}$ refers to both the soliton and the uniform background. Therefore, in order to obtain the "pure" soliton momentum, the background contribution must be subtracted. As long as we follow the scheme from Ref. [13], the background part of the momentum $\mathcal{P}_{S}^{(u)}$ is obtained as

$$
\mathcal{P}_{S}^{(u)}\left(v_{S}\right)=-\kappa_{\infty}\left[\delta \theta\left(v_{S}\right)-\delta \theta(0)\right],
$$

where $\kappa_{\infty} \equiv \kappa\left(a_{\infty}\right)$ with $a_{\infty} \equiv 1, \delta \theta\left(v_{S}\right)$ is the total change of the phase determined by (26) and (27), which depends on the soliton velocity, and $\delta \theta(0)=\pi$. Performing the subtraction of the background contribution, we arrive at the result

$$
\mathcal{P}_{S}\left(v_{S}\right)=v_{S} \int_{-\infty}^{\infty} d x \frac{\left[\kappa(a) a^{2}-\kappa_{\infty}\right]^{2}}{\rho_{\mathrm{sf}}(a)}-\pi \kappa_{\infty} .
$$

We use the replacement of the integration variable and solution for the amplitude, (32). The resulting soliton momentum is then given by

$$
\mathcal{P}_{S}\left(v_{S}\right)=2 \sqrt{2} v_{S} \int_{a_{0}}^{1} \frac{\sqrt{\rho_{\mathrm{qp}}(a)} Y(a)}{\sqrt{X(a)-v_{S}^{2} Y(a)}} d a-\pi \kappa_{\infty} .
$$

It is easy to check that the subtraction of $\pi \kappa_{\infty}$ in (39) is necessary in order to ensure the zero-velocity limit $\lim _{v_{S} \rightarrow 0} \mathcal{P}_{S}\left(v_{S}\right)=0$.

The soliton energy is defined accordingly to the rule of the classical mechanics:

$$
\mathcal{E}_{S} \equiv v_{S} \mathcal{P}_{S}-\mathcal{L}=\mathcal{H}
$$

Using the exact solutions for the amplitude and the phase, we arrive at a simple expression for the energy:

$$
\mathcal{E}_{S}\left(v_{S}\right)=2 \int_{-\infty}^{\infty} d x\left[\Omega_{s}(a)-\Omega_{s}\left(a_{\infty}\right)\right] .
$$

As for the momentum, the replacement of variables ( $a$ instead of $x$ ) yields the soliton energy expressed through the integral over the amplitude modulation:

$$
\mathcal{E}_{S}\left(v_{S}\right)=2 \sqrt{2} \int_{a_{0}}^{1} \frac{\sqrt{\rho_{\mathrm{qp}}(a)} X(a)}{\sqrt{X(a)-v_{S}^{2} Y(a)}} d a .
$$

Next, we check whether relation (49) is fulfilled for a dark soliton within the present GL-like approach. The derivative $\partial \mathcal{E}_{S} / \partial \mathcal{P}_{S}$ can be expressed as

$$
\frac{\partial \mathcal{E}_{S}}{\partial \mathcal{P}_{S}}=\left(\frac{\partial \mathcal{P}_{S}}{\partial v_{S}}\right)^{-1}\left(\frac{\partial \mathcal{E}_{S}}{\partial v_{S}}\right)
$$

The details of the calculation for the derivatives are represented in the Appendix. Here, we represent the final results:

$$
\begin{aligned}
\frac{\partial \mathcal{E}_{S}}{\partial v_{S}}= & 2 \sqrt{2} v_{S} \int_{a_{0}}^{1}\left(\frac{\sqrt{\rho_{\mathrm{qp}}(a)} X(a) Y(a)}{\left[X(a)-Y(a) v_{S}^{2}\right]^{3 / 2}}\right. \\
& \left.-\frac{\sqrt{\rho_{\mathrm{qp}}\left(a_{0}\right)} X\left(a_{0}\right) Y\left(a_{0}\right)}{\left[G\left(a_{0}\right)\right]^{3 / 2}\left(a-a_{0}\right)^{3 / 2}}\right) d a \\
& -4 \sqrt{2} v_{S} \frac{\sqrt{\rho_{\mathrm{qp}}\left(a_{0}\right)} X\left(a_{0}\right) Y\left(a_{0}\right)}{\left[G\left(a_{0}\right)\right]^{3 / 2} \sqrt{1-a_{0}}}
\end{aligned}
$$

and

$$
\begin{aligned}
\frac{\partial \mathcal{P}_{S}}{\partial v_{S}}= & 2 \sqrt{2} \int_{a_{0}}^{1}\left(\frac{\sqrt{\rho_{\mathrm{qp}}(a)} X(a) Y(a)}{\left[X(a)-Y(a) v_{S}^{2}\right]^{3 / 2}}\right. \\
& \left.-\frac{\sqrt{\rho_{\mathrm{qp}}\left(a_{0}\right)} X\left(a_{0}\right) Y\left(a_{0}\right)}{\left[G\left(a_{0}\right)\right]^{3 / 2}\left(a-a_{0}\right)^{3 / 2}}\right) d a \\
& -4 \sqrt{2} \frac{\sqrt{\rho_{\mathrm{qp}}\left(a_{0}\right)} X\left(a_{0}\right) Y\left(a_{0}\right)}{\left[G\left(a_{0}\right)\right]^{3 / 2} \sqrt{1-a_{0}}},
\end{aligned}
$$

where the function $G(a)$ is given by formula (A4).

The effective mass of the dark soliton can be introduced as in Ref. [13]:

$$
M_{S} \equiv \frac{\partial \mathcal{P}_{S}}{\partial v_{S}} .
$$

Therefore formula (45) allows us to determine the effective mass of the dark soliton explicitly:

$$
\begin{aligned}
M_{S}\left(v_{S}\right)= & 2 \sqrt{2} \int_{a_{0}}^{1}\left(\frac{\sqrt{\rho_{\mathrm{qp}}(a)} X(a) Y(a)}{\left[X(a)-Y(a) v_{S}^{2}\right]^{3 / 2}}\right. \\
& \left.-\frac{\sqrt{\rho_{\mathrm{qp}}\left(a_{0}\right)} X\left(a_{0}\right) Y\left(a_{0}\right)}{\left[G\left(a_{0}\right)\right]^{3 / 2}\left(a-a_{0}\right)^{3 / 2}}\right) d a \\
& +\frac{4 \sqrt{2} \sqrt{\rho_{\mathrm{qp}}\left(a_{0}\right)} X\left(a_{0}\right) Y\left(a_{0}\right)}{\left[G\left(a_{0}\right)\right]^{3 / 2} \sqrt{1-a_{0}}} .
\end{aligned}
$$

Comparing (A8) with (A9) we find the exact analytic relation:

$$
\frac{\partial \mathcal{E}_{S}}{\partial v_{S}}=v_{S} \frac{\partial \mathcal{P}_{S}}{\partial v_{S}} .
$$

The identity (43) combined with (48) gives us the same equation as in Ref. [13]:

$$
\frac{\partial \mathcal{E}_{S}}{\partial \mathcal{P}_{S}}=v_{S}
$$

This equation shows that the soliton described within the present formalism obeys the classic Hamilton dynamics, i.e., moves like a particle. This behavior holds even in the most general case-for arbitrary $\Omega_{s}(a)$ and amplitude-dependent coefficients in the effective field action.

\section{RESULTS AND DISCUSSION}

The subsequent numerical analysis is restricted to the case of a balanced Fermi gas, where the populations of the "spin-up" and "spin-down" fermions are equal. The soliton parameters are calculated here in two approximations: (i) within the saddle-point approximation for $\left|\Psi_{\infty}\right|$ and the chemical potential $\mu$, which are obtained using the mean-field number and gap equations; and (ii) accounting for fluctuations about the saddle point. The Gaussian fluctuations are included here within the same scheme as in Refs. [24] and [36], through the renormalization of the chemical potential of the Fermi gas. In both cases, the results obtained within our EFT are compared with results obtained with BdG theory applied at unitarity, from Ref. [21]. 

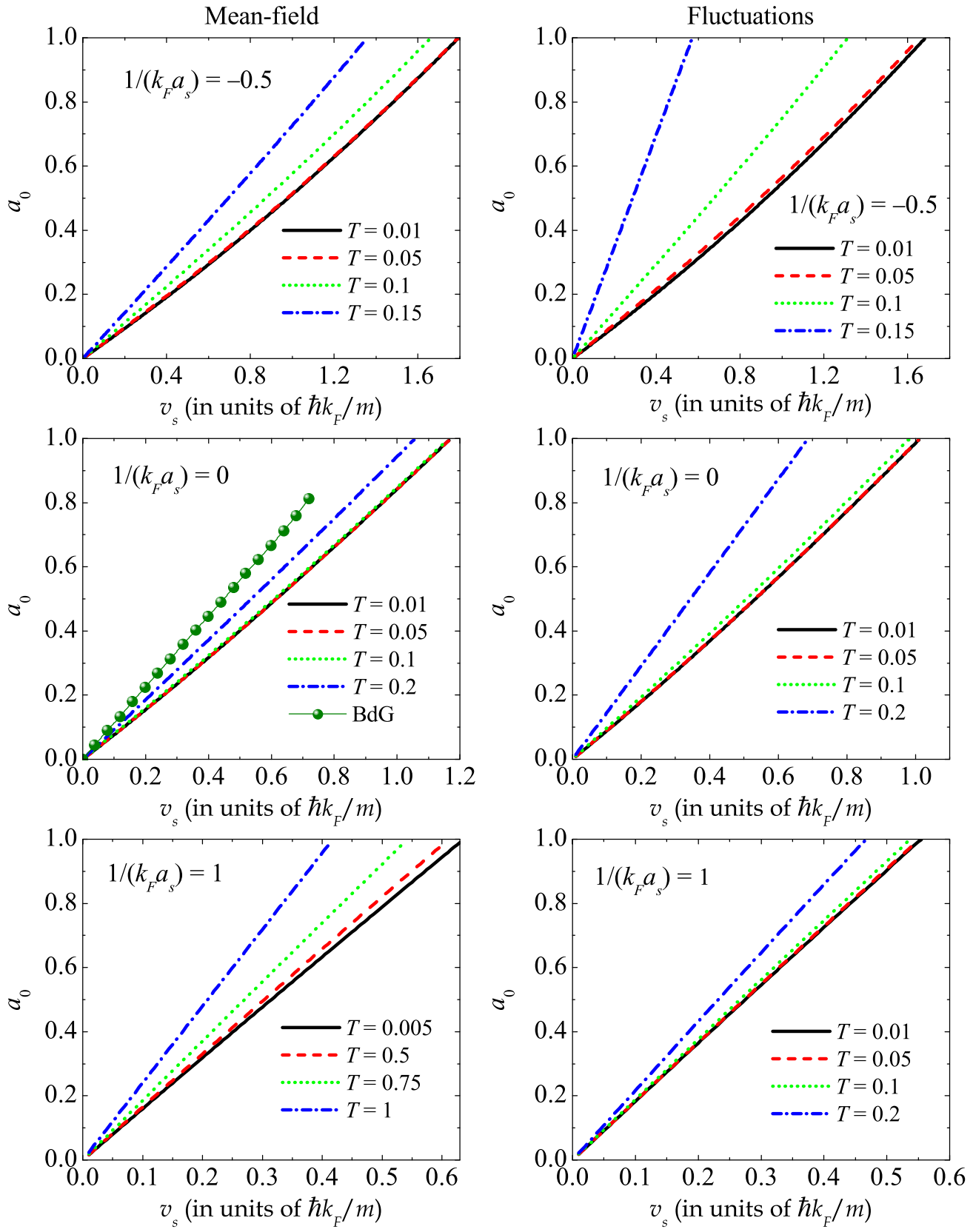

FIG. 1. (Color online) Amplitude modulation function at the soliton center dependent on the soliton velocity $v_{S}$ for different scattering lengths and temperatures. Left panels: Mean-field calculation. Right panels: Results obtained accounting for Gaussian fluctuations. Symbols (filled circles) show the results of the BdG theory from Ref. [21].

It should be noted that the effective action for the pair field, (1), has been derived in Ref. [35] using a gradient expansion up to second order in spatial gradients and in imaginary time gradients. This is consistent with the assumption that the pair field slowly varies in space and time. Consequently, keeping the coordinate and time dependence of the coefficients $C$ and $E$ that appear in front of the second-order gradient factors is, strictly speaking, beyond the second order and may lead to artifacts in the limiting case when simultaneously $T \rightarrow 0$ and $a \rightarrow 0$. Therefore in the present numerical analysis we keep the coefficients $C(a)$ and $E(a)$ equal to their background (bulk) values $C\left(a_{\infty}\right)$ and $E\left(a_{\infty}\right)$. On the contrary, in the thermodynamic potential $\Omega_{s}(a)$ and in the first-order terms of the gradient expansion in the effective action (time derivatives), we keep the amplitude dependence of the coefficients.

The results are presented in Figs. 1-8. Each figureexcept Fig. 3-shows how a solitonic property depends on the soliton velocity $v_{S}$ and is divided into six panels. The top row contains results for the BCS regime $\left(a_{s}=-0.5\right)$; the middle row, for unitarity $\left(a_{s}=0\right)$; and the bottom row, for the BEC regime $\left(a_{s}=1\right)$. The left column shows the results using the mean-field value for $\left|\Psi_{\infty}\right|, \mu$, and the right column shows the results including fluctuations in $\left|\Psi_{\infty}\right|, \mu$. Filled circles in the figures represent BdG results from Ref. [21]. 

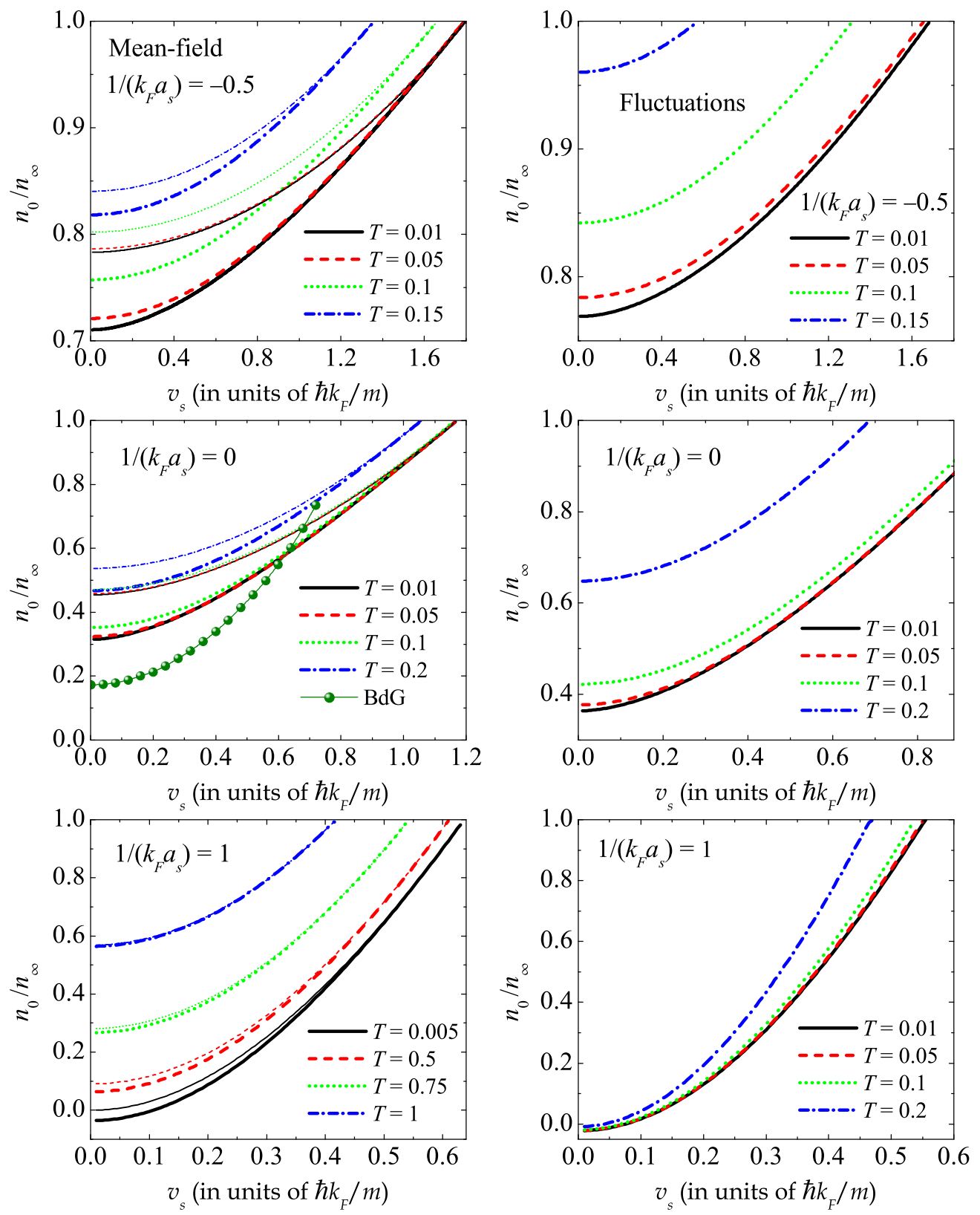

FIG. 2. (Color online) Relative fermion density at the soliton center $n_{0} / n_{\infty}$ as a function of the soliton velocity $v_{S}$ for different scattering lengths and temperatures. Thick curves: Density calculated accounting for gradient terms. Thin curves: Density calculated within the LDA. Filled circles show the results of the BdG theory from Ref. [21].

Figure 1 shows the ratio of the amplitude of the order parameter $\Psi$ at the soliton center to the bulk value $\left|\Psi_{\infty}\right|$ [in other words, the amplitude modulation function $\left.a(x)\right|_{x=0} \equiv a_{0}$ ] as a function of the soliton velocity $v_{S}$. The dependence $a_{0}\left(v_{S}\right)$ is close to a linear function for all considered temperatures and scattering lengths. The slope of that linear dependence rises with increasing temperature. The critical velocity $v_{S}^{(c)}$, when $a_{0}=1$, indicates a breakdown of the soliton state: a soliton does not exist for $v_{S}>v_{S}^{(c)}$. The critical velocity obtained in the present work is close to the sound velocity determined in Ref. [21] for the unitarity regime as $c=v_{F} \sqrt{\mu /\left(3 E_{F}\right)}$, where $v_{F}$ is the Fermi velocity (equal to $v_{F}=2$ in the present units). The critical velocity diminishes when the temperature rises. The obtained close-to-linear dependence of $a_{0}\left(v_{S}\right)$ is in line with the results of the BdG theory from Ref. [21] but increases slightly more slowly than the BdG solution in the unitarity regime.

In Fig. 2, we plot the relative fermion density dip at the soliton center $n_{0} / n_{\infty}$ (where $n_{\infty}$ is the bulk fermion density) as a function of the soliton velocity for the same initial parameters as in Fig. 1. It is clear that even though the pair density at the soliton center may become low (as $v_{S} \rightarrow 0$ ), the soliton partially fills up with unpaired atoms, leading to $n_{0} / n_{\infty}>a_{0}$. The fermion density is determined here in two ways: (i) within the mean-field local density approximation (LDA), using the formula

$$
n^{(\mathrm{LDA})}=-\frac{\partial \Omega_{s}}{\partial \mu}
$$




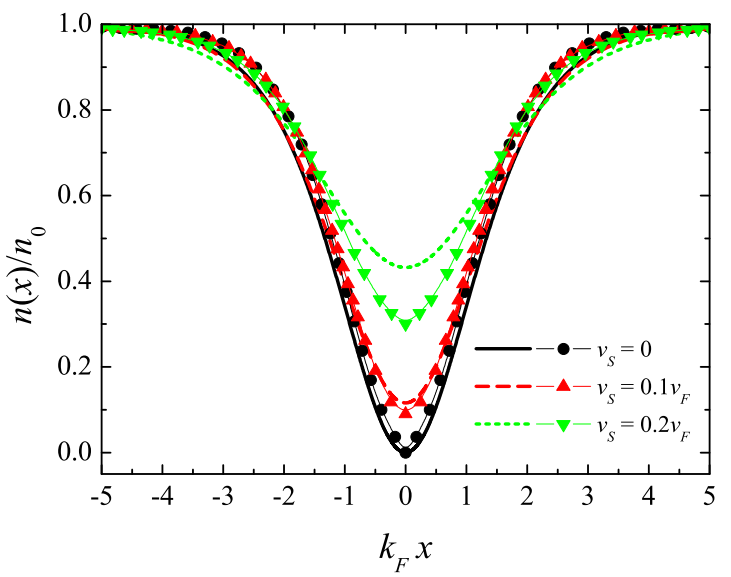

FIG. 3. (Color online) The fermion density profile $n_{x} / n_{\infty}$ of the dark soliton as a function of the distance to the soliton center $(x=0)$ is shown for several soliton velocities at $1 /\left(k_{F} a_{s}\right)=1$ and compared with BdG results (filled circles).

and (ii) accounting for the "gradient part" of the densityprovided by the gradient terms in the Hamiltonian, (17),

$$
n^{\text {(grad) }}=-\frac{\partial \Omega_{s}}{\partial \mu}-\frac{1}{2} \frac{\partial \rho_{\mathrm{qp}}}{\partial \mu}(\nabla a)^{2}-\frac{1}{2} \frac{\partial \rho_{\mathrm{sf}}}{\partial \mu}(\nabla \theta)^{2} .
$$

Comparing the graphs for $n_{0} / n_{\infty}$ with different scattering lengths, we see that the relative contribution of the gradient part of the density is more significant for weaker coupling strengths and for lower temperatures: the highest difference between $n^{\text {(grad) }}$ and $n^{(\mathrm{LDA})}$ occurs in the BCS regime at the lowest considered temperature. The relative depth of the fermion density dip qualitatively follows the BdG results, being slightly smaller at $v_{S}=0$ and showing a less expressed dependence on the soliton velocity with respect to the BdG data.

The gradient correction in the fermion density in (51) is a kind of perturbation. Therefore $n^{\text {(grad) }}$ is reliable when the gradient term is relatively small with respect to the LDA expression. This is the case everywhere except the soliton center in the BEC regime, when $n^{(\mathrm{LDA})}$ becomes extremely small at $x=0$. Consequently, (51) can result in an artifact in the BEC regime: a low but negative density near the soliton center. Thus $n^{(\mathrm{LDA})}$ is more suitable than $n^{\text {(grad) }}$ on the BEC side. However, at unitarity and in the BCS regime, $n^{\text {(grad) }}$ provides a closer agreement of the density profile with respect to the BdG data.

Figure 3 shows the overall density profile of the soliton, as a function of the distance from the center of the soliton, calculated using formula (50). Also, this is seen to reproduce the BdG results well. However, in the BCS regime, the Friedel oscillations of the density obtained in the BdG calculations do not agree with the results in the current formalism, even though the profile closer to $x=0$ is still the same in both formalisms.

The disagreement between BdG and EFT results in the BCS regime can be explained as follows. The key approximation of the EFT is the local gradient expansion. This expansion is a common ingredient of the EFT and several other approaches, e.g., the GL formalism and the recently developed coarsegraining approach for the BdG equations [23]. Already in early works $[38,39]$, it was pointed out that the range of validity of the local gradient expansion must be restricted by the condition that the pair field is slowly varying over distances characterizing the spatial extent of the electron pair correlation (the coherence length). In the BCS regime, the coherence length can be associated with the Cooper pair size. The comparison of the results of the local gradient expansion with the BdG data for a vortex performed in Ref. [23] explicitly confirmed the expectation that a local gradient expansion can fail in the BCS regime at low temperature, when the size of the Cooper pairs is quite large and comparable to that of the vortex. As a result, the gradient expansion can fail at low temperatures in the BCS regime. On the contrary, on the BEC side the Fermi gas turns to a Bose gas of molecules, which is well described by a GP-like equation.

In the present work, we compare the parameters of a dark soliton obtained within the EFT with the BdG results for $T=0$, because at present we do not possess BdG data for dark solitons for nonzero temperatures. However, the comparison of the EFT and BdG results for a finite-temperature vortex performed in Ref. [34] confirms the aforesaid reasoning regarding the range of validity of the local gradient expansion. First, a good agreement with the BdG theory [23] is found except in the BCS regime at low temperatures. Second, the agreement of EFT with BdG becomes gradually better when the temperature rises from 0 to $T_{c}$. Moreover, as shown analytically in [34], the EFT exactly retrieves the GL approach in the vicinity of $T_{c}$.

Figure 4 shows the total phase difference $\delta \theta$ through the soliton. The comparison with the BdG results is performed for the unitarity regime. As for the density, the $\mathrm{BdG}$ calculation gives a faster decrease in the total phase change as a function of $v_{S}$ with respect to that calculated using EFT. As stated in Ref. [21], one of the key results of the BdG approach applied to dark solitons is a drastic qualitative difference of the phase difference with respect to that obtained using the GP equation. The GP method yields $\cos \left[\delta \theta\left(v_{S}\right)\right] \propto v_{S}$, so that at low velocities one obtains $\pi-\delta \theta\left(v_{S}\right) \propto v_{S}^{2}$. On the contrary, the $\mathrm{BdG}$ approach results in a linear dependence $\delta \theta\left(v_{S}\right)$, at least at small $v_{S}$. Within the EFT, $\delta \theta\left(v_{S}\right)$ is linear at low velocities, which is more realistic with respect to the GP results and closer to the BdG data. However, a quantitative difference between the present results and the BdG results at unitarity remains.

In Fig. 5, the soliton energy $E_{S}\left(v_{S}\right)$ is plotted and compared with the BdG data for all three regimes (BCS, unitarity, and BEC). The best coincidence of the soliton energy calculated within the BDG and EFT methods is obtained in the BEC regime. At weaker couplings, the maximum of the energy (at $v_{S}=0$ ) provided by the $\mathrm{BdG}$ method is higher than that obtained within EFT. At low velocities, the energy is approximately quadratic with a negative second derivative, indicating a negative effective mass of the soliton. The soliton energy falls to 0 when the velocity reaches its critical value $v_{S}^{(c)}$. Figure 6 shows the soliton momentum $P_{S}\left(v_{S}\right)$. At small $v_{S}$, the dependence $P_{S}\left(v_{S}\right)$ is close to a linear function with a negative slope (which also indicates a negative effective mass of the soliton). With increasing velocity, the soliton momentum ends at a finite value when $v_{S}$ reaches a critical value $v_{S}^{(c)}$ (different for different coupling strengths and temperatures). The energymomentum relation, (49), has been numerically checked in the 

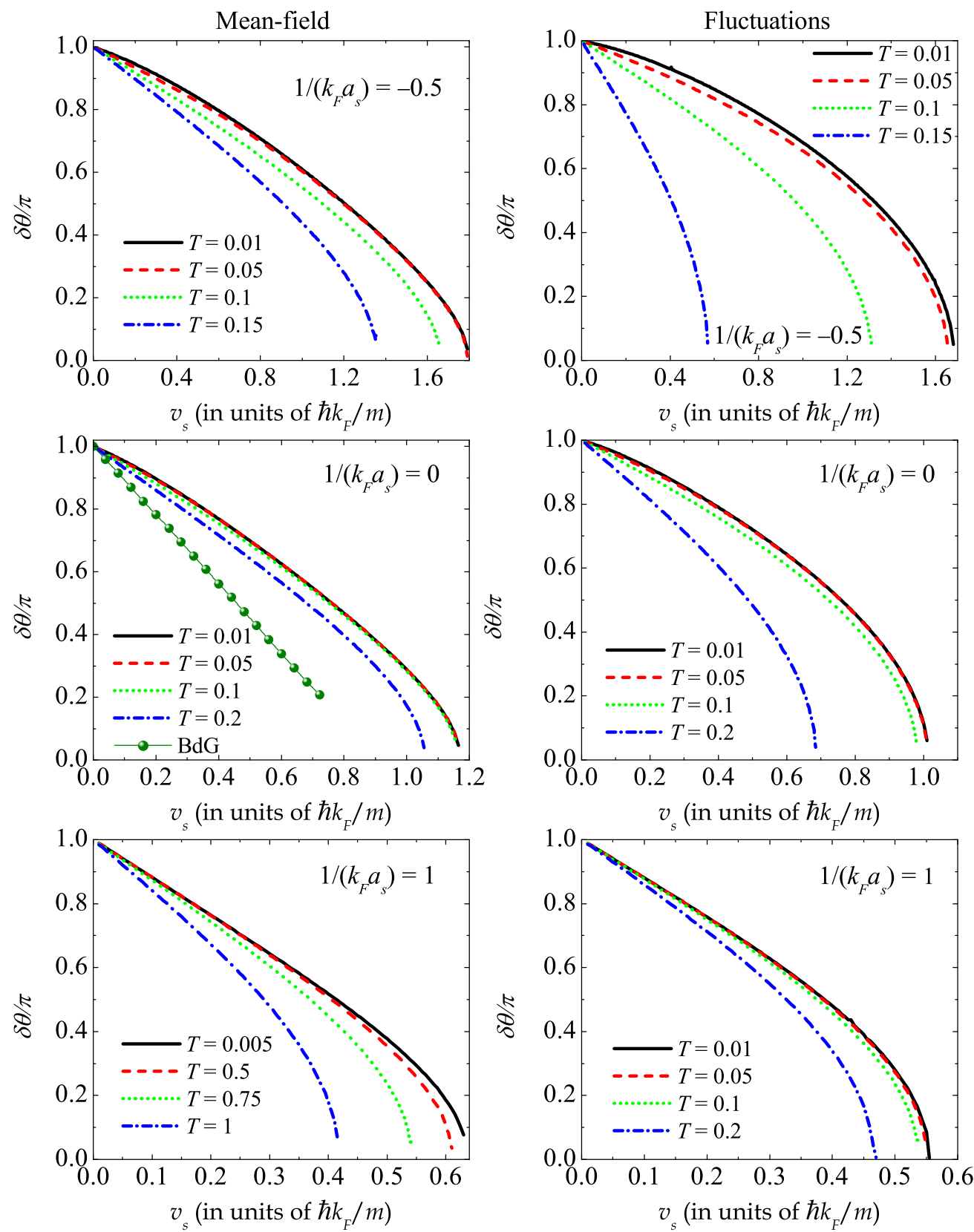

FIG. 4. (Color online) Phase difference $\delta \theta$ as a function of the soliton velocity $v_{S}$. Notation is the same as in Figs. 1 and 2.

present calculation for the data represented in Figs. 5 and 6. This verification has shown that it is indeed fulfilled.

In Fig. 7, we plot the number of fermions in the soliton cloud $N_{S}$ (per unit area in the $y z$ plane), determined by the integral

$$
N_{S}=\int_{-\infty}^{\infty}[n(x)-n(\infty)] d x
$$

When multiplied by the fermion mass $m$ (here, $m=1 / 2$ as in our previous calculations), the fermion number yields the "physical mass" of a soliton $m N_{S}$ [21]. The fermion density in a dark soliton is lower than the bulk fermion density. Hence the number of fermions $N_{S}$, as well as the physical mass of a dark soliton, is negative. The absolute number of fermions $\left|N_{S}\right|$ in a soliton monotonously decreases as a function of the velocity $v_{S}$. The absolute value $\left|N_{S}\right|$ gradually rises with increasing coupling strength and diminishes with increasing temperature. The comparison with the $\mathrm{BdG}$ data [21] is possible at present for the unitarity regime. At low velocities, the BdG and EFT results for the number of fermions in the soliton match each other very well. The increase in the number of fermions in the soliton as a function of $v_{S}$ is, however, faster for the $\mathrm{BdG}$ method than for the EFT.

Finally, Fig. 8 represents the effective mass of the soliton determined by formulas (46) and (47). As follows from (49), the other definition of the effective mass (e.g., in Ref. [21]),

$$
M_{S}=\frac{1}{v_{S}} \frac{\partial \mathcal{E}_{S}}{\partial v_{S}},
$$



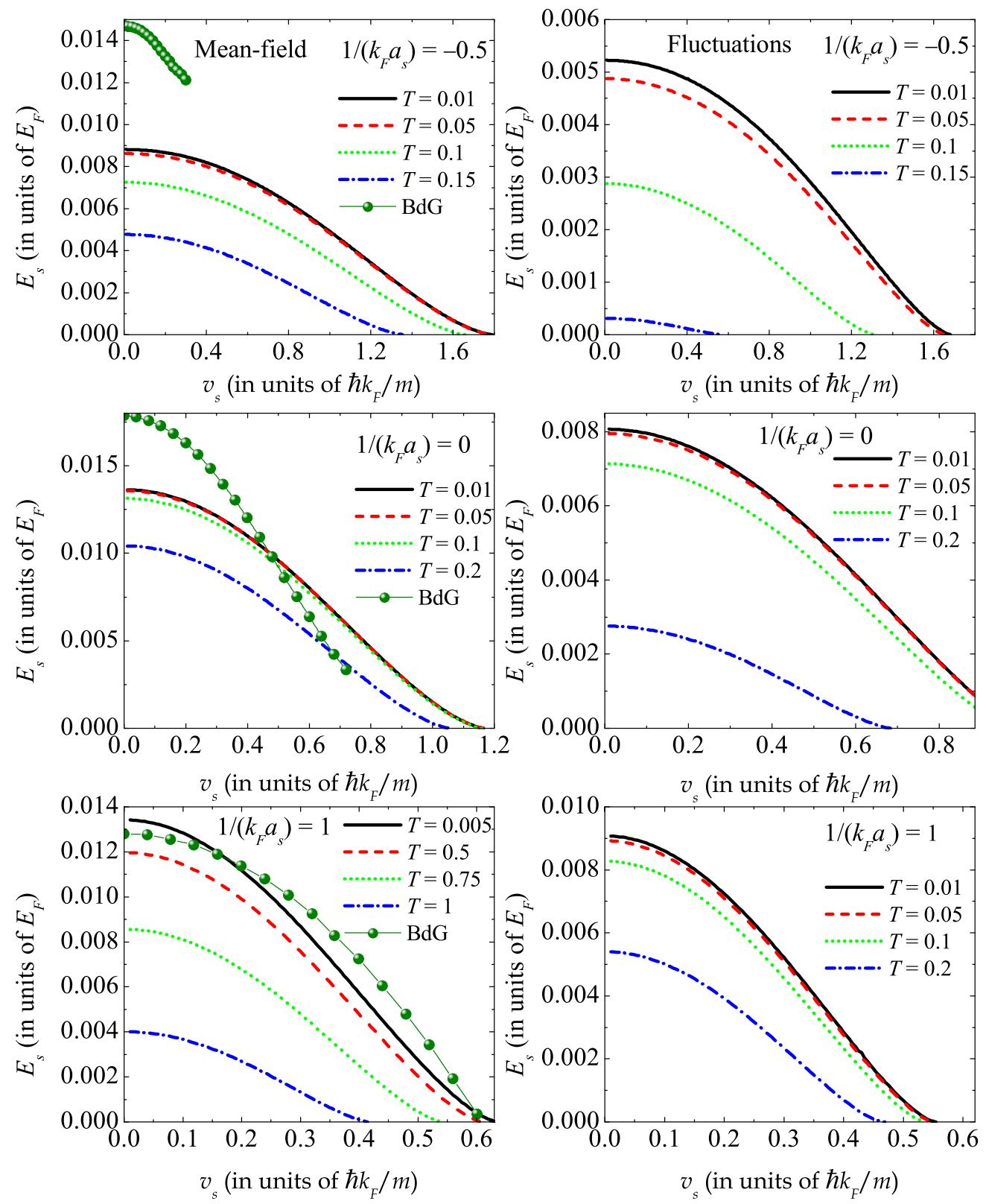

FIG. 5. (Color online) Soliton energy $\mathcal{E}_{S}\left(v_{S}\right)$ as a function of the soliton velocity $v_{S}$. Filled circles represent BdG data [21].

is equivalent to (47). The effective and physical masses of a dark soliton are, in general, different [40]. The effective mass in the BCS and unitarity regimes nonmonotonously behaves as a function of velocity. In the BEC regime, however, $\left|M_{S}\right|$ monotonously decreases when $v_{S}$ rises. At unitarity and at weak coupling, the effective and physical masses are rather close to each other. In the BEC regime the effective mass of the soliton appears to be larger (in absolute value) than the physical mass.

The comparison of the soliton parameters calculated with and without the effect of the Gaussian fluctuations shows that the range of the soliton velocities $v_{S}<v_{S}^{(c)}$ where the soliton exists is relatively slightly influenced by the fluctuations in all three regimes: in the $\mathrm{BCS}$ regime $\left(1 / a_{s}=-0.5\right)$, at unitarity $\left(1 / a_{s}=0\right)$, and in the $\mathrm{BEC}$ regime $\left(1 / a_{s}=1\right)$. The behavior of the amplitude modulation function, the fermion density, and the phase difference exhibits the same trend. On the contrary, the relative change of the soliton energy, the soliton momentum, and both the physical and the effective masses is rescaled more strongly: even in the BCS regime it is not small. Qualitatively, the dependence of the calculated dark soliton parameters on the soliton velocity, the temperature, and the coupling strength is similar to that obtained within the mean-field approach. The effect of the fluctuations consists in a scaling of the calculated parameters due to the renormalization of the density. The critical temperature for a Fermi gas obtained accounting for fluctuations is lower than the meanfield critical temperature, especially at sufficiently strong coupling (at unitarity and in the BEC regime, where $T_{c}$ with fluctuations tends to a constant value when $1 / a_{s} \rightarrow+\infty$, contrary to the mean-field critical temperature). Consequently, the dark soliton parameters in the BEC regime calculated 

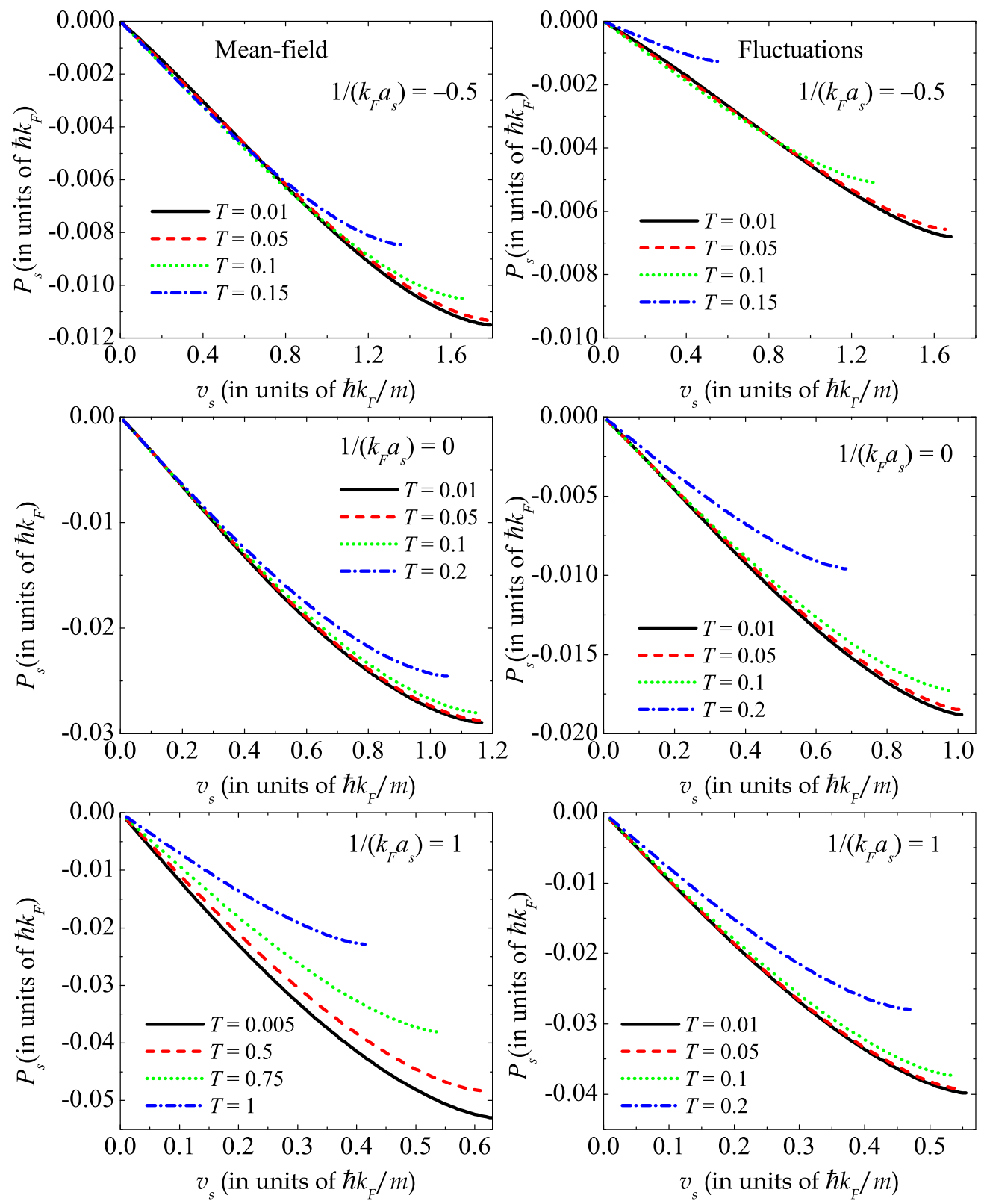

FIG. 6. (Color online) Soliton momentum $\mathcal{P}_{S}\left(v_{S}\right)$ as a function of the soliton velocity $v_{S}$.

accounting for fluctuations are significantly more sensitive to temperature than those calculated within the mean-field approximation.

\section{CONCLUSIONS}

Within the EFT, we have derived the analytic solution of the field equations which describes a dark soliton in a superfluid Fermi gas with $s$-wave pairing for arbitrary temperatures below $T_{c}$ and for arbitrary values of the inverse scattering length, encompassing the BCS-BEC crossover regime. The macroscopic parameters of the dark soliton (modulus of the order parameter and phase profile) are analytically expressed by assuming that the order parameter has the usual solitonic $f\left(x-v_{s} t\right)$ time dependence, expressing the conservation of the soliton shape as it travels at constant speed. This assumption does not allow us to investigate the decay of the soliton due to the snake instability [9-12] and does not take into account the inhomogeneity or anisotropy due to trapping, even though the EFT itself allows, in principle, to investigate this decay dynamics as well. Here, we compute the density profile and, with it, the filling of the soliton "core" by unpaired fermions. The exact energy-momentum relation for the soliton has been derived, showing that the soliton as a whole obeys classical Hamiltonian dynamics, with a well-determined effective mass, dependdent on the velocity.

The GL theory in its original form was developed as an expansion of the free energy of a superconductor in powers of the order parameter near $T_{c}$. Therefore it is valid, strictly speaking, only in the close vicinity of the critical temperature. The finite-temperature EFT is a straightforward extension 

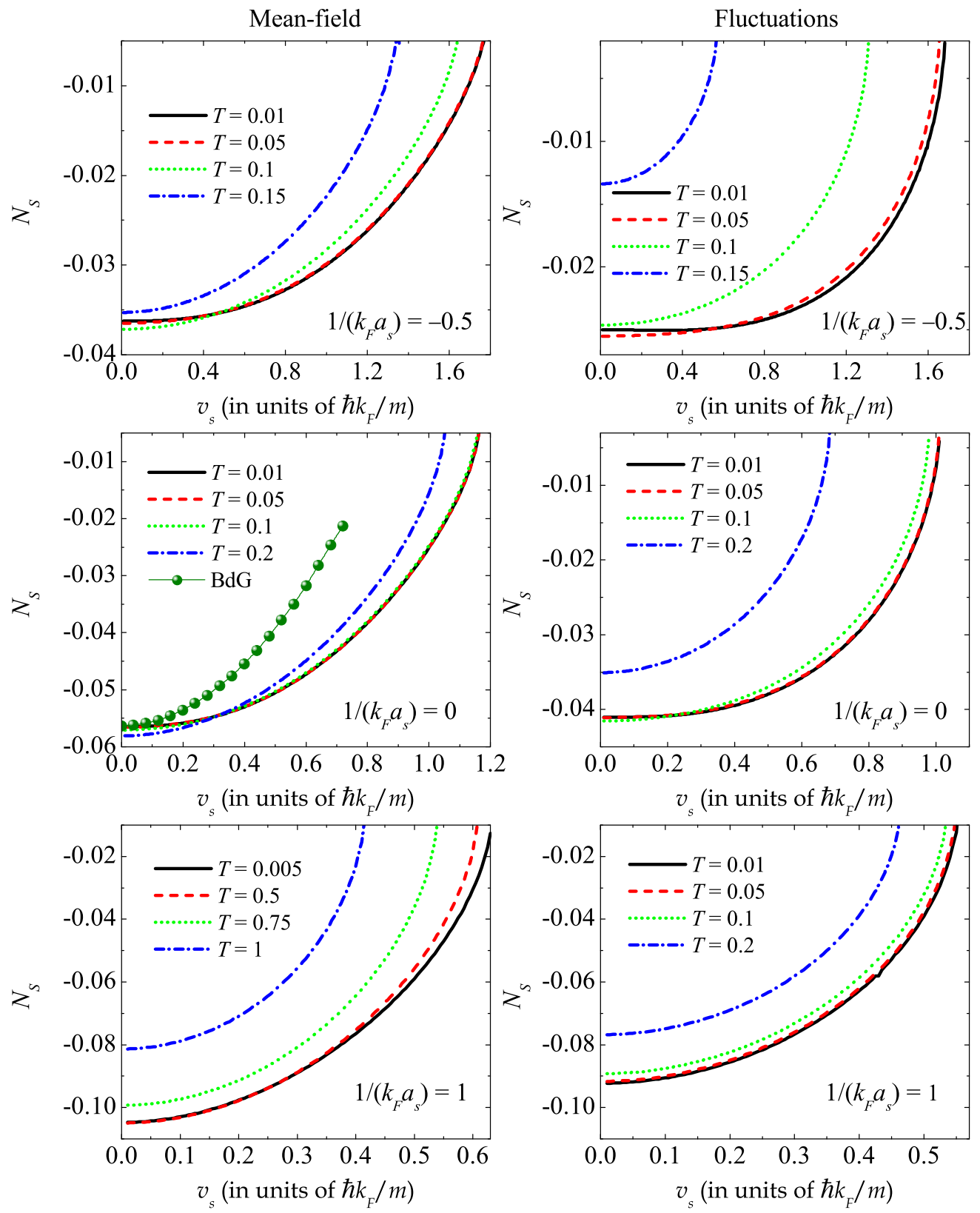

FIG. 7. (Color online) Number of fermions in the soliton $N_{S}\left(v_{S}\right)$ as a function of the soliton velocity $v_{S}$. Filled circles represent BdG results from Ref. [21].

of the GL method beyond this restriction, for the whole temperature range below $T_{c}$, retrieving the GL theory as a limiting case when $T \rightarrow T_{c}$. This allows us to compare the parameters of dark solitons with the corresponding $\mathrm{BdG}$ results at 0 temperature, where the GL method is not valid. We find good agreement except in the deep BCS regime. For solitons in Fermi gases, at present no $T>0 \mathrm{BdG}$ results are available. However, the validity of our method was checked for vortices. In [34], we find that the results of the present formalism agree well with BdG results, and the agreement improves as $T$ goes to $T_{c}$. Near $T_{c}$, the result of the GL formalism in Ref. [24] is retrieved. This indicates a substantial extension of the range of validity of the present method with respect to the standard GL. Therefore, despite the lack of finite-temperature BdG data for solitons in Fermi gases, we expect that the agreement between
EFT and BdG will be better for nonzero temperatures than for $T=0$.

Besides the mean-field calculation, we have taken into account the Gaussian fluctuations through the renormalization of the chemical potential of the fermions. This renormalization keeps the qualitative picture of the soliton similar to that obtained within the mean-field approximation but leads to quantitative changes of the soliton parameters and distributions.

\section{ACKNOWLEDGMENTS}

We are grateful to J. Brand, L. Salasnich, and G. C. Strinati for valuable discussions. This work was supported by the Flemish Research Foundation (FWO-V1), Projects No. G.0370.09N, No. G.0180.09N, No. G.0115.12N, No. 

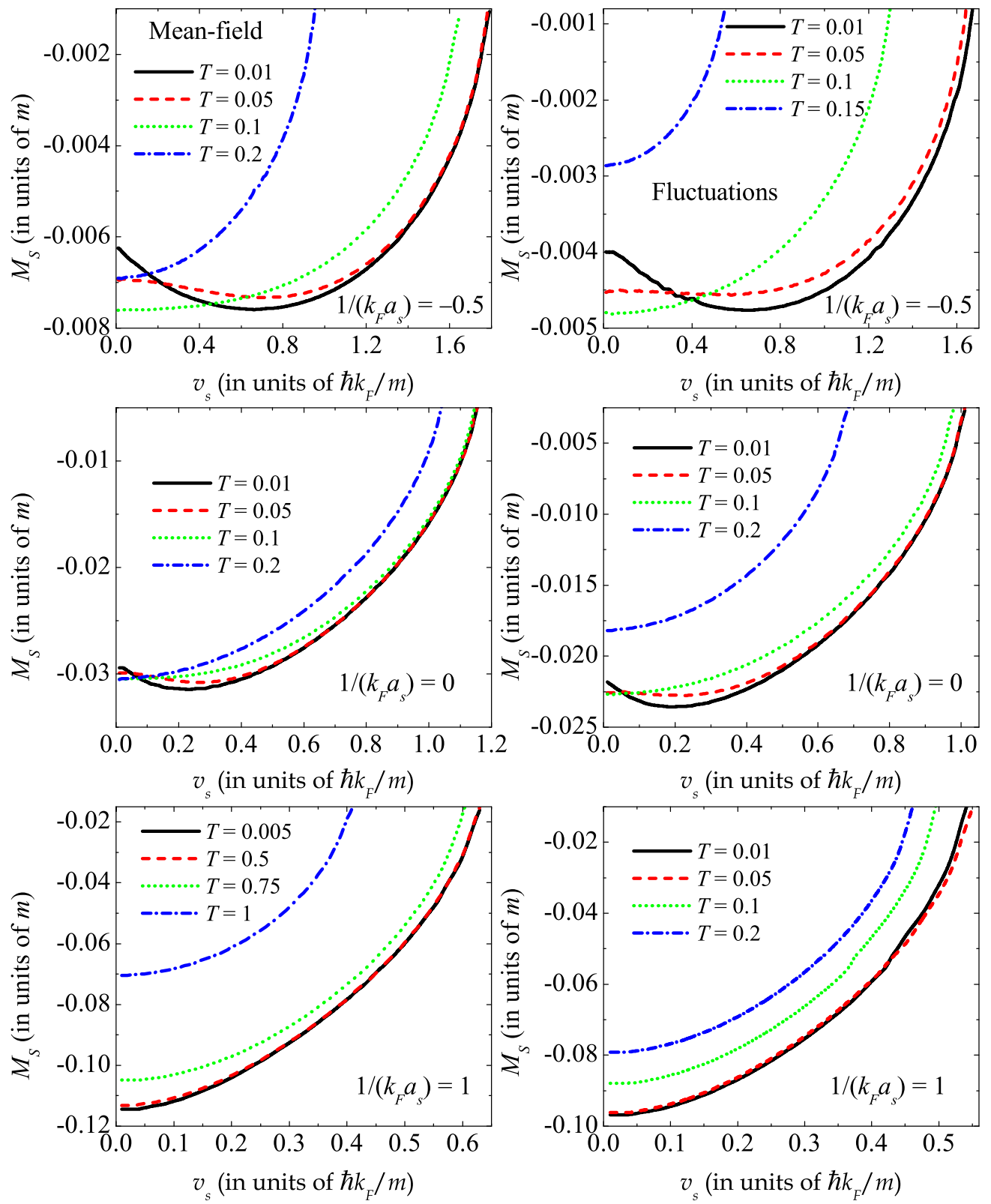

FIG. 8. (Color online) Effective mass of the soliton $M_{S}\left(v_{S}\right)$ as a function of the soliton velocity $v_{S}$.

G.0119.12N, and No. G.0122.12N, and by the Scientific Research Network of the Research Foundation-Flanders, WO.033.09N, and by the Research Council of Antwerp University, project DOCPRO 2014 - 29778.

\section{APPENDIX: DERIVATIVES OF THE ENERGY AND THE MOMENTUM}

When we take the derivative $\partial \mathcal{E}_{S} / \partial v_{S}$ in (42) straightforwardly, the integral over $a$ becomes divergent at $a \rightarrow a_{0}$ due to the appearance of the factor $\left[X(a)-v_{S}^{2} Y(a)\right]^{3 / 2}$ in the denominator. To remove divergencies, we consider the auxiliary expression with the parameter $\delta>0$ :

$$
\mathcal{E}_{S}\left(v_{S}, \delta\right) \equiv 2 \sqrt{2} \int_{a_{0}}^{1} \frac{\sqrt{\rho_{\mathrm{qp}}(a)} X(a)}{\sqrt{X(a)-v_{S}^{2} Y(a)+\delta}} d a .
$$

The function $\mathcal{E}_{S}\left(v_{S}, \delta\right)$ turns to $\mathcal{E}_{S}\left(v_{S}\right)$ in the limit $\delta \rightarrow+0$.

Differentiating $\mathcal{E}_{S}\left(v_{S}, \delta\right)$ with respect to $v_{S}$, we obtain the result

$$
\begin{aligned}
\frac{\partial \mathcal{E}_{S}\left(v_{S}, \delta\right)}{\partial v_{S}}= & 2 \sqrt{2} v_{S} \int_{a_{0}}^{1} \frac{\sqrt{\rho_{\mathrm{qp}}(a)} X(a) Y(a)}{\left[X(a)+\delta-Y(a) v_{S}^{2}\right]^{3 / 2}} d a \\
& -\frac{\partial a_{0}}{\partial v_{S}} \frac{2 \sqrt{2}}{\sqrt{\delta}} \sqrt{\rho_{\mathrm{qp}}\left(a_{0}\right)} X\left(a_{0}\right) .
\end{aligned}
$$

The regularization of (A2) is found using the Taylor series of the denominator about the point $a=a_{0}$. Accounting for (33), we find the expansion of the function $X(a)-v_{S}^{2} Y(a)+\delta$ about $a_{0}$ :

$$
X(a)-v_{S}^{2} Y(a)+\delta=G\left(a_{0}\right)\left(a-a_{0}\right)+\delta+\cdots,
$$


where we have denoted the function

$$
G(a) \equiv \frac{\partial X(a)}{\partial a}-v_{S}^{2} \frac{\partial Y(a)}{\partial a}
$$

Next, consider the auxiliary integral,

$$
\int_{a_{0}}^{1} \frac{1}{\left(G \cdot\left(a-a_{0}\right)+\delta\right)^{3 / 2}} d a=2 \frac{-\sqrt{\delta}+\sqrt{G-G a_{0}+\delta}}{\sqrt{G-G a_{0}+\delta} G \sqrt{\delta}} .
$$

In the limit of small $\delta$, this integral behaves as $1 / \sqrt{\delta}$ :

$$
\int_{a_{0}}^{1} \frac{1}{\left(G \cdot\left(a-a_{0}\right)+\delta\right)^{3 / 2}} d a=\frac{2}{\sqrt{\delta} G}-\frac{2}{G^{3 / 2} \sqrt{1-a_{0}}}+O(\delta) .
$$

Thus we can express the factor $2 /(G \sqrt{\delta})$ as

$$
\frac{2}{G \sqrt{\delta}}=\int_{a_{0}}^{1} \frac{1}{\left(G \cdot\left(a-a_{0}\right)+\delta\right)^{3 / 2}} d a+\frac{2}{G^{3 / 2} \sqrt{1-a_{0}}}+O(\delta) .
$$

The factor $\partial a_{0} /\left(\partial v_{S}\right)$ is determined as follows:

$$
\frac{\partial a_{0}}{\partial v_{S}}=-\frac{\frac{\partial\left(X\left(a_{0}\right)-Y\left(a_{0}\right) v_{S}^{2}\right)}{\partial v_{S}}}{\frac{\partial\left(X\left(a_{0}\right)-Y\left(a_{0}\right) v_{S}^{2}\right)}{\partial a_{0}}}=2 v_{S} \frac{Y\left(a_{0}\right)}{G\left(a_{0}\right)} .
$$

Using these results the derivative $\partial \mathcal{E}_{S}(\delta) / \partial v_{S}$ is transformed to the expression

$$
\frac{\partial \mathcal{E}_{S}(\delta)}{\partial v_{S}}=2 \sqrt{2} v_{S} \int_{a_{0}}^{1}\left(\frac{\sqrt{\rho_{\mathrm{qp}}(a)} X(a) Y(a)}{\left[X(a)-v_{S}^{2} Y(a)+\delta\right]^{3 / 2}}-\frac{\sqrt{\rho_{\mathrm{qp}}\left(a_{0}\right)} X\left(a_{0}\right) Y\left(a_{0}\right)}{\left[G\left(a_{0}\right)\left(a-a_{0}\right)+\delta\right]^{3 / 2}}\right) d a-4 \sqrt{2} v_{S} \frac{\sqrt{\rho_{\mathrm{qp}}\left(a_{0}\right)} X\left(a_{0}\right) Y\left(a_{0}\right)}{\left[G\left(a_{0}\right)\right]^{3 / 2} \sqrt{1-a_{0}}} .
$$

The resulting integral over $a$ converges. Hence we can explicitly set $\delta \rightarrow 0$ in (A7), yielding the regularized expression for the derivative $\partial \mathcal{E}_{S} / \partial v_{S}$ :

$$
\frac{\partial \mathcal{E}_{S}}{\partial v_{S}}=2 \sqrt{2} v_{S} \int_{a_{0}}^{1}\left(\frac{\sqrt{\rho_{\mathrm{qp}}(a)} X(a) Y(a)}{\left[X(a)-Y(a) v_{S}^{2}\right]^{3 / 2}}-\frac{\sqrt{\rho_{\mathrm{qp}}\left(a_{0}\right)} X\left(a_{0}\right) Y\left(a_{0}\right)}{\left[G\left(a_{0}\right)\right]^{3 / 2}\left(a-a_{0}\right)^{3 / 2}}\right) d a-4 \sqrt{2} v_{S} \frac{\sqrt{\rho_{\mathrm{qp}}\left(a_{0}\right)} X\left(a_{0}\right) Y\left(a_{0}\right)}{\left[G\left(a_{0}\right)\right]^{3 / 2} \sqrt{1-a_{0}}} .
$$

Repeating the same steps for the derivative of the momentum, we arrive at the regularized expression:

$$
\frac{\partial \mathcal{P}_{S}}{\partial v_{S}}=2 \sqrt{2} \int_{a_{0}}^{1}\left(\frac{\sqrt{\rho_{\mathrm{qp}}(a)} X(a) Y(a)}{\left[X(a)-Y(a) v_{S}^{2}\right]^{3 / 2}}-\frac{\sqrt{\rho_{\mathrm{qp}}\left(a_{0}\right)} X\left(a_{0}\right) Y\left(a_{0}\right)}{\left[G\left(a_{0}\right)\right]^{3 / 2}\left(a-a_{0}\right)^{3 / 2}}\right) d a-4 \sqrt{2} \frac{\sqrt{\rho_{\mathrm{qp}}\left(a_{0}\right)} X\left(a_{0}\right) Y\left(a_{0}\right)}{\left[G\left(a_{0}\right)\right]^{3 / 2} \sqrt{1-a_{0}}} .
$$

[1] M. H. Anderson, J. R. Ensher, M. R. Matthews et al., Science 269, 198 (1995).

[2] K. B. Davis, M. O. Mewes, M. R. Andrews, N. J. van Druten, D. S. Durfee, D. M. Kurn, and W. Ketterle, Phys. Rev. Lett. 75, 3969 (1995).

[3] S. Burger, K. Bongs, S. Dettmer, W. Ertmer, K. Sengstock, A. Sanpera, G. V. Shlyapnikov, and M. Lewenstein, Phys. Rev. Lett. 83, 5198 (1999).

[4] J. Denschlag, J. E. Simsarian, D. L. Feder, C. W. Clark, L. A. Collins, J. Cubizolles, L. Deng, E. W. Hagley, K. Helmerson, W. P. Reinhardt, S. L. Rolston, B. I. Schneider, and W. D. Phillips, Science 287, 97 (2000).

[5] Z. Chen, M. Segev, and D. N. Christodoulides, Rep. Prog. Phys. 75, 086401 (2012).

[6] B. Denardo, B. Galvin, A. Greenfield, A. Larraza, S. Putterman, and W. Wright, Phys. Rev. Lett. 68, 1730 (1992).

[7] A. Chabchoub, O. Kimmoun, H. Branger, N. Hoffmann, D. Proment, M. Onorato, and N. Akhmediev, Phys. Rev. Lett. 110, 124101 (2013).
[8] T. Yefsah, A. T. Sommer, M. J. H. Ku, L. W. Cheuk, W. Ji, W. S. Bakr, and M. W. Zwierlein, Nature 499, 426 (2013).

[9] A. Cetoli, J. Brand, R. G. Scott, F. Dalfovo, and L. P. Pitaevskii, Phys. Rev. A 88, 043639 (2013).

[10] A. Bulgac, M. M. Forbes, M. M. Kelley, K. J. Roche, and G. Wlazłowski, Phys. Rev. Lett. 112, 025301 (2014).

[11] W. Wen, C. Zhao, and X. Ma, Phys. Rev. A 88, 063621 (2013).

[12] L. A. Toikka and K.-A. Suominen, Phys. Rev. A 87, 043601 (2013).

[13] D. J. Frantzeskakis, J. Phys. A 43, 213001 (2010).

[14] D. L. Feder, M. S. Pindzola, L. A. Collins, B. I. Schneider, and C. W. Clark, Phys. Rev. A 62, 053606 (2000).

[15] B. Jackson, C. F. Barenghi, and N. P. Proukakis, J. Low Temp. Phys. 148, 387 (2007).

[16] B. Jackson, N. P. Proukakis, and C. F. Barenghi, Phys. Rev. A 75, 051601 (2007).

[17] A. D. Martin and J. Ruostekoski, Phys. Rev. Lett. 104, 194102 (2010). 
[18] J. Dziarmaga, Z. P. Karkuszewski, and K. Sacha, J. Phys. B: At. Mol. Opt. Phys. 36, 1217 (2003).

[19] D. M. Gangardt and A. Kamenev, Phys. Rev. Lett. 104, 190402 (2010).

[20] A. Spuntarelli, P. Pieri, and G. C. Strinati, Phys. Rep. 488, 111 (2010).

[21] R. Liao and J. Brand, Phys. Rev. A 83, 041604 (2011).

[22] A. Spuntarelli, L. D. Carr, P. Pieri, and G. C. Strinati, New J. Phys. 13, 035010 (2011).

[23] S. Simonucci and G. C. Strinati, Phys. Rev. B 89, 054511 (2014).

[24] C. A. R. Sa de Melo, M. Randeria, and J. R. Engelbrecht, Phys. Rev. Lett. 71, 3202 (1993).

[25] K. Huang, Z.-Q. Yu, and L. Yin, Phys. Rev. A 79, 053602 (2009).

[26] S. K. Adhikari, Phys. Rev. A 77, 045602 (2008).

[27] A. A. Shanenko, M. V. Milosevic, F. M. Peeters, and A. V. Vagov, Phys. Rev. Lett. 106, 047005 (2011).

[28] A. Vagov, A. A. Shanenko, M. V. Milošević, V. M. Axt, and F. M. Peeters, Phys. Rev. B 86, 144514 (2012).
[29] A. Chaves, L. Komendová, M. V. Milošević, J. S. Andrade Jr., G. A. Farias, and F. M. Peeters, Phys. Rev. B 83, 214523 (2011).

[30] N. V. Orlova, A. A. Shanenko, M. V. Milošević, F. M. Peeters, A. V. Vagov, and V. M. Axt, Phys. Rev. B 87, 134510 (2013).

[31] E. Babaev and M. Speight, Phys. Rev. B 72, 180502 (2005).

[32] E. Babaev, J. Carlstrom, and M. Speight, Phys. Rev. Lett. 105, 067003 (2010).

[33] E. Babaev and M. Silaev, Phys. Rev. B 86, 016501 (2012).

[34] S. N. Klimin, J. Tempere, and J. T. Devreese, arXiv:1309.1421.

[35] S. N. Klimin, J. Tempere, and J. T. Devreese, Physica C 503, 136 (2014).

[36] J. Tempere, S. N. Klimin, and J. T. Devreese, Phys. Rev. A 78, 023626 (2008).

[37] E. Taylor, A. Griffin, N. Fukushima, and Y. Ohashi, Phys. Rev. A 74, 063626 (2006).

[38] L. Tewordt, Phys. Rev. 132, 595 (1963).

[39] N. R. Werthammer, Phys. Rev. 132, 663 (1963).

[40] R. G. Scott, F. Dalfovo, L. P. Pitaevskii, and S. Stringari, Phys. Rev. Lett. 106, 185301 (2011). 